OPEN ACCESS

Edited by:

Youjun Liu,

Beijing University of Technology, China

Reviewed by:

Shengzhang Wang,

Fudan University, China

Zengsheng Chen,

Beihang University, China

*Correspondence:

Yong Luan

cclyyly@163.com

Kairong Qin

krqin@dlut.edu.cn

Specialty section:

This article was submitted to Computational Physiology and

Medicine,

a section of the journal

Frontiers in Physiology

Received: 30 June 2021

Accepted: 20 August 2021

Published: 21 September 2021

Citation:

Wang Y, Wang J, Peng J, Huo M, Yang Z, Giridharan GA, Luan Y and

Qin K (2021) Effects of a Short-Term

Left Ventricular Assist Device on

Hemodynamics in a Heart Failure

Patient-Specific Aorta Model: A CFD

Study. Front. Physiol. 12:733464

doi: 10.3389/fphys.2021.733464

\section{Effects of a Short-Term Left Ventricular Assist Device on Hemodynamics in a Heart Failure Patient-Specific Aorta Model: A CFD Study}

\author{
Yu Wang ${ }^{1}$, Junwei Wang ${ }^{1}$, Jing Peng ${ }^{1}$, Mingming Huo ${ }^{1}$, Zhiqiang Yang ${ }^{2}$, \\ Guruprasad A. Giridharan ${ }^{3}$, Yong Luan ${ }^{4 *}$ and Kairong Qin ${ }^{1 *}$
}

'School of Optoelectronic Engineering and Instrumentation Science, Dalian University of Technology, Dalian, China, ${ }^{2}$ Department of Cardiovascular Computed Tomography (CT) Examination, The First Affiliated Hospital of Dalian Medical University, Dalian, China, ${ }^{3}$ Department of Bioengineering, University of Louisville, Louisville, KY, United States, ${ }^{4}$ Department of Anesthesiology, The First Affiliated Hospital of Dalian Medical University, Dalian, China

Patients with heart failure (HF) or undergoing cardiogenic shock and percutaneous coronary intervention require short-term cardiac support. Short-term cardiac support using a left ventricular assist device (LVAD) alters the pressure and flows of the vasculature by enhancing perfusion and improving the hemodynamic performance for the HF patients. However, due to the position of the inflow and outflow of the LVAD, the local hemodynamics within the aorta is altered with the LVAD support. Specifically, blood velocity, wall shear stress, and pressure difference are altered within the aorta. In this study, computational fluid dynamics (CFD) was used to elucidate the effects of a short-term LVAD for hemodynamic performance in a patient-specific aorta model. The three-dimensional (3D) geometric models of a patient-specific aorta and a short-term LVAD, Impella CP, were created. Velocity, wall shear stress, and pressure difference in the patient-specific aorta model with the Impella CP assistance were calculated and compared with the baseline values of the aorta without Impella CP support. Impella CP support augmented cardiac output, blood velocity, wall shear stress, and pressure difference in the aorta. The proposed CFD study could analyze the quantitative changes in the important hemodynamic parameters while considering the effects of Impella CP, and provide a scientific basis for further predicting and assessing the effects of these hemodynamic signals on the aorta.

Keywords: CFD, hemodynamic performance, patient-specific aorta model, left ventricular assist device, heart failure

\section{INTRODUCTION}

Cardiovascular diseases are the leading cause of death and they account for $56 \%$ of total mortality in the modern era. Among such diseases, the number of patients with heart failure (HF) has exceeded 20 million worldwide, and is increasing at an annual rate of about 2 million people (Benjamin et al., 2017). HF refers to the clinical syndrome of circulatory disorder caused by the obstruction 
of systolic and/or diastolic functions of the heart, which cannot fully discharge the amount of venous return to the heart (Doehner et al., 2018; Van der Meer et al., 2019). HF is not an independent disease, but a chronic and progressive disease and the end stage of the development of cardiovascular diseases (Koshy et al., 2020; McEwan et al., 2020). Therefore, ventricular geometry transition can be observed associated with the endstage HF (Li et al., 2020; Guan et al., 2021; Shavik et al., 2021). Moreover, hemodynamic variables such as flow pattern, wall shear stress (WSS), and distribution of blood pressure difference $(\Delta P)$ in the aorta could also be aberrant with the development of HF, causing vascular and arterial remodeling (Nakamura, 1999; Ky et al., 2013; Leite et al., 2019), and eventually inducing vascular and aortic diseases such as aorta coarctation, aortic valve stenosis, aortic stiffness, aortic insufficiency, etc. (Reddy et al., 2017; Nagao et al., 2018; Keen and Johnson, 2019). Therefore, quantitatively studying hemodynamic behaviors in the aorta regarding the geometric structure and functional characteristics is of great significance for the diagnosis and treatment of HF.

Furthermore, since HF is generally not curable but treatable, left ventricular assist devices (LVADs), as life-saving medical devices, have been successfully developed for the HF treatment (Slaughter et al., 2009; Schumer et al., 2016; Rogers et al., 2017). Durable LVAD support has been initiated in patients with chronic HF as it is superior to optimal medical management. Although heart transplantation has the best outcomes, there is an extreme lack of availability of heart donors in the world (Simaan et al., 2009). In addition, patients who are under acute cardiogenic shock or patients with imminent HF due to coronary heart disease are provided with short-term LVAD as a bridge to a decision or hemodynamic support during the percutaneous coronary intervention (PCI; i.e., stents) to mitigate cardiac hypoxia. LVADs can enhance perfusion and generally improve hemodynamic status by lowering the left ventricular work and increasing aortic pressure and flow. However, the local hemodynamic effects of the LVADs on aorta, especially the fluid velocity profile, WSS, and $\Delta P$, are altered due to the outlet of the LVAD. Thus, it would be necessary to numerically analyze hemodynamics of the aorta for patients implanted with the LVADs.

To analyze hemodynamics in the aorta quantitatively, analyzing the fluid-dynamic variables is essential. However, traditional strategies using lumped parameter models using in vitro even in vivo studies are not adequate to fully understand geometric and functional properties and fluid-dynamic of the aorta (Gramigna et al., 2015; Carnahan et al., 2017; Wang et al., 2017). A crucial challenge facing such studies is that usually high fidelity pressure and/or flow measurements for hemodynamic analysis are extremely difficult because of the complex geometry and small dimensions of the aortic arch and blood vessels (Mazzitelli et al., 2016). These measurements also require implantation of sensors, which require invasive thoracotomy or sternotomy and is not feasible in patients due to the higher risk of mortality. Therefore, the hemodynamics in the aorta was investigated via computational fluid dynamics (CFD).

Different CFD; models have been established to quantitatively analyze the hemodynamics in the aorta under varying physiological conditions. For instance, studies by coupling CFD and some medical imaging technologies like Doppler echocardiography and magnetic resonance imaging (MRI) effectively predicted aneurysm and coarctation of the aorta (Brüning et al., 2018; Perinajová et al., 2021). Compared with MRI or computed tomography (CT), some expected dynamic behaviors of aortic diseases under stressed conditions can be better assessed via CFD modeling (Osswald et al., 2017; Febina et al., 2018), which can also be used to analyze the formation mechanism of aortic diseases (Zhang X. et al., 2020). However, most HF-related studies did not model the effects of LVAD support on the aorta. There have been literature studies demonstrating the changes in hemodynamics due to LVAD support using lumped parameter models, but these models are incapable of elucidating the local effects of the LVAD support. CFD is necessary to obtain the local distribution of some key parameters such as WSS, velocity vectors, etc. A few CFD studies combining the aorta and LVADs were based on the long-term, durable, and surgically-implanted LVADs (Mazzitelli et al., 2016; Yoshida et al., 2020), which have different outlets and outflows compared with short-term percutaneously inserted devices. Furthermore, short-term LVADs are used for patients with different indications compared with durable LVADs. For instance, Impella, one class of short-term LVADs, is a microaxial and catheter-mounted blood pump, which is percutaneously inserted into the aorta. Impella is indicated for circulatory support to patients with cardiogenic shock and high-risk PCI (Schrage et al., 2019; Amin et al., 2020), who do not always have significant remodeling of the heart and aorta due to prolonged and chronic HF. Therefore, the hemodynamic effects of the aorta in conjunction with a short-term blood pump need to be further studied.

In this work, the CFD simulation is implemented using a patient-specific aorta model by adding the effects of a shortterm blood pump (Impella CP), in order to better understand the local hemodynamic effects in the aorta. Velocity, WSS, and $\Delta P$ distribution in the aorta are analyzed and also compared with the results only with the patient-specific aorta model without Impella CP support.

\section{MATERIALS AND METHODS}

\section{Governing Equations}

In this CFD simulation, blood is assumed to be the incompressible Newtonian fluid. The governing equations for blood flows are set as follows (Deissler, 1984):

$$
\begin{aligned}
& \frac{\partial \bar{V}_{i}}{\partial t}+\bar{V}_{j} \frac{\partial \bar{V}_{i}}{\partial x_{j}}=-\frac{1}{\rho} \frac{\partial \bar{p}}{\partial x_{i}}+\frac{\mu}{\rho} \frac{\partial^{2} \bar{V}_{i}}{\partial x_{i} \partial x_{j}}-\frac{\overline{\partial V_{i}^{\prime} V_{j}^{\prime}}}{\partial x_{j}} \\
& \frac{\partial \bar{V}_{i}}{\partial x_{i}}=0
\end{aligned}
$$

where $\bar{V}_{i}(i=1,2,3)$ represents the average velocities, $t$ is the time, $x_{i}$ represents the space coordinates in three-dimensional Cartesian coordinate system, $\mu$ is the dynamic viscosity at 0.004 $\mathrm{kg} / \mathrm{m}^{-1} / \mathrm{s}^{-1}, \rho$ is the fluid density at $1,060 \mathrm{~kg} / \mathrm{m}^{3}, \bar{p}$ is the average 
pressure. The second item on the right side in Equation (1) reflects the viscous effect, shear stress, and $\bar{\tau}_{i j}$, can be rewritten as:

$$
\bar{\tau}_{i j}=\mu \frac{\partial \bar{V}_{i}}{\partial x_{j}}
$$

The last term in Equation (1) is called Reynolds stress term. Since this term is unknown, the shear stress transport $k-\omega$ turbulence model is used for the CFD simulation (Menter, 1994). The turbulent kinetic energy transport equation and specific dissipation rate transport equation are as follows (Qiao et al., 2016):

$$
\begin{gathered}
\rho \frac{\partial k}{\partial t}+\rho \frac{\partial\left(k \bar{V}_{i}\right)}{\partial x_{i}}=\frac{\partial}{\partial x_{j}}\left(\Gamma_{k} \frac{\partial k}{\partial x_{j}}\right)+G_{k}-Y_{k} \\
\rho \frac{\partial \omega}{\partial t}+\rho \frac{\partial\left(\omega \bar{V}_{i}\right)}{\partial x_{i}}=\frac{\partial}{\partial x_{j}}\left(\Gamma_{\omega} \frac{\partial \omega}{\partial x_{j}}\right)+G_{\omega}-Y_{\omega}
\end{gathered}
$$

where $k$ and $\omega$ represent the turbulent scalar, called turbulent kinetic energy and specific dissipation rate, respectively. $G_{k}$ is the turbulent kinetic energy produced by laminar velocity gradient, $G_{\omega}$ is the turbulent kinetic energy generated by equation (5). $\Gamma_{k}$ and $\Gamma_{\omega}$ represent the effective diffusion terms of $k$ and $\omega$, respectively; $Y_{k}$ and $Y_{\omega}$ represent turbulence due to the diffusion.

\section{Geometric Model of the Patient-Specific Aorta}

The enhanced-CT medical image data used in this study were from The First Affiliated Hospital of Dalian Medical University, China. The subject was an 80-year-old man with valve disease and reduced cardiac output. For the image data, the layer spacing is $0.74 \mathrm{~mm}$, and the plane resolution is $512 \times 512$. This enhanced CT image data were imported into Mimics 21.0 (Materialise, Leuven, Belgium), a medical image processing software, with DICOM (digital imaging and communications in medicine) format for data processing and three-dimensional (3D) reconstruction, to acquire a geometric model of the patient-specific aorta. Automatic threshold segmentation and manual separation were used to separate the ascending aorta, brachiocephalic trunk, left common carotid artery, left subclavian artery, descending aorta, and abdominal aorta from other tissues. After smooth processing, the 3D reconstruction model of the aortic arch was obtained while maintaining the original physiological and anatomical characteristics, shown in Figure 1A.

\section{Geometry of Impella CP}

The 3D structure model of Impella CP drawn with a 3D software, Proe 2.0 (Parametric Technology Corporation, Boston, MA, USA), crosssection mesh structure of Impella CP, and the location of Impella $\mathrm{CP}$ with its partially enlarged detail are shown in Figure 2. The pump body is an impeller, composed of a shaft with a fixed end and double blades with free end, and rotates counterclockwise during operation. Two blades are generated by spiral scanning and parameterization, and the impeller is enclosed in the pump housing. The two bottoms of the cylinder were considered as the inlet and outlet of the housing, respectively. Blood was pumped from the left ventricle into the housing inlet, and flows out of the outlet. Note that the specific geometric sizes and parameters of Impella $\mathrm{CP}$ are referred from the literature (Roberts et al., 2020).

\section{Patient-Specific Aorta Model Without the Impella CP Support}

The 3D geometric model of the patient-specific aorta was imported into the commercial CFD software package, ANSYS Fluent 2020 R1 (Ansys Inc., Canonsburg, PA, USA) for mesh generation. The domain tetrahedral unstructured meshes of about 850,000 elements were established, partly shown in Figures $\mathbf{3 A}, \mathbf{B}$, and the boundary layer was set on the surfaces to meet the constraints of the turbulence model. The fluid part of the grid is imported into ANSYS Fluent 2020 R1 to set boundary conditions. Pulsatile blood velocity at the inlet of aorta is considered as the inlet condition, and the change of blood flow velocity with time is shown in Figure 3C. Note that the velocity waveform used as the inlet condition in this study was obtained from the literature (Estrada et al., 2011). It was scaled in order to make it match the physiological characteristics in the human cardiovascular system such that the baseline flow rate without Impella $\mathrm{CP}$ support was less than $3 \mathrm{~L} / \mathrm{min}$ (Sayago et al., 2015). It is assumed that at the inlet of aorta, the distribution of blood flow velocity, $V_{i n}$, on the crosssection of blood vessels perpendicular to the direction of blood flow is parabolic, whose contour is shown in Figure 3D, and the blood flow velocity in the polar coordinate system is expressed as:

$$
V_{\text {in }}(r, t)=2 V_{\text {in_mean }}(t)\left[1-\left(\frac{2 r}{d}\right)^{2}\right]
$$

Note that since the inlet surface is approximately a circular surface, the centroid and area of the inlet surface can be obtained using ANSYS Fluent $2020 \mathrm{R} 1$, and the centroid is regarded as the center of an approximate circular surface. Therefore, in Equation (6), $r$ represents the distance from the approximate center and $d$ represents the approximate diameter calculated based on the area of inlet surface, $\boldsymbol{V}_{\text {in_mean }}$ is the average velocity on the crosssection. The above mathematical expressions for calculating the required waveform of blood flow velocity was written in C-language with user-defined functions (UDFs), which can be compiled and loaded into the fluent library. Zero pressure at the outlet of aorta is prescribed as the outlet condition (Zhang J. et al., 2020).

In addition, at present, it is difficult to obtain blood flow data in the three branches of aorta such as brachiocephalic trunk, left common carotid artery, and left subclavian artery on the basis of the individual patients with various cardiovascular diseases. Therefore, in this study, the sum of blood flow from the above three branches accounted for 30\% (Chi et al., 2017) out of the total flow rates. In the meanwhile, the ratio of flow rates from brachiocephalic trunk, left common carotid artery, and left subclavian artery was set as 1.5:1:1.2 (Chi et al., 2017), respectively. As a result, the blood flow velocity 
A

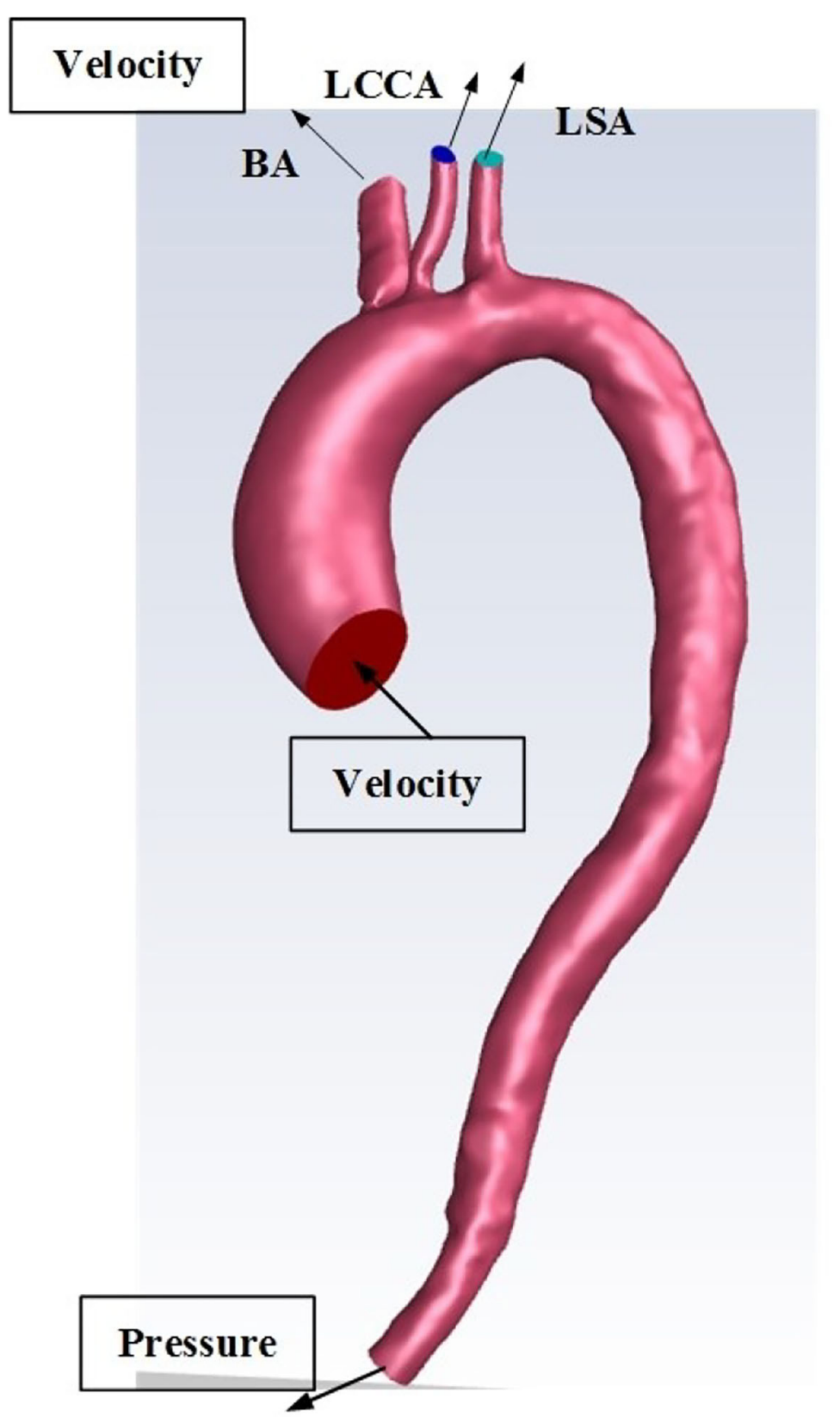

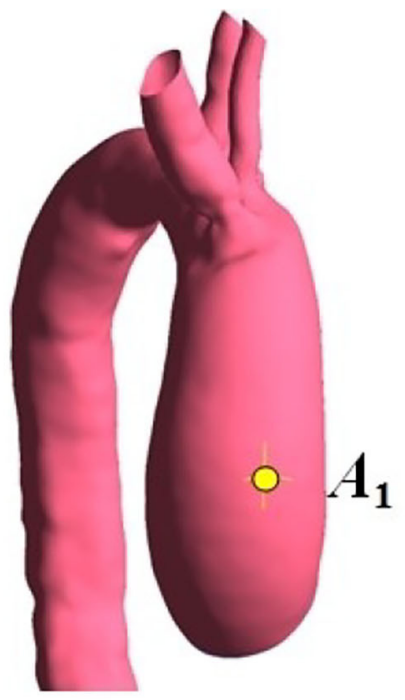

C

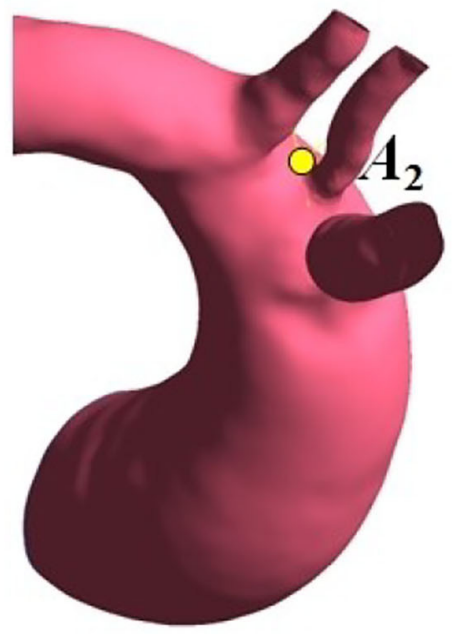

FIGURE 1 | (A) Reconstructed geometry of the aortic arch for an HF patient. (B) selected location $A_{1}$. (C) selected location $A_{2}$. BA, brachiocephalic trunk; LCCA, left common carotid artery; LSA, left subclavian artery.

of brachiocephalic trunk, left common carotid artery, and left subclavian artery can be calculated based on the crosssection areas of the above three branch outlets. In addition, while the three branches are set as velocity inlets, they have a negative inlet velocity to represent the outflows out of the three arch vessels (Hu et al., 2020). Furthermore, in this study, we assumed that there were no additional branching vessels in the aorta downstream of the three main aortic branches to simplify the CFD simulation.

\section{Patient-Specific Aorta Model During the Impella CP Support}

First, after using Proe 2.0 to establish the $3 \mathrm{D}$ geometric model of Impella CP, ANSYS Fluent 2020 R1 is used to mesh the 

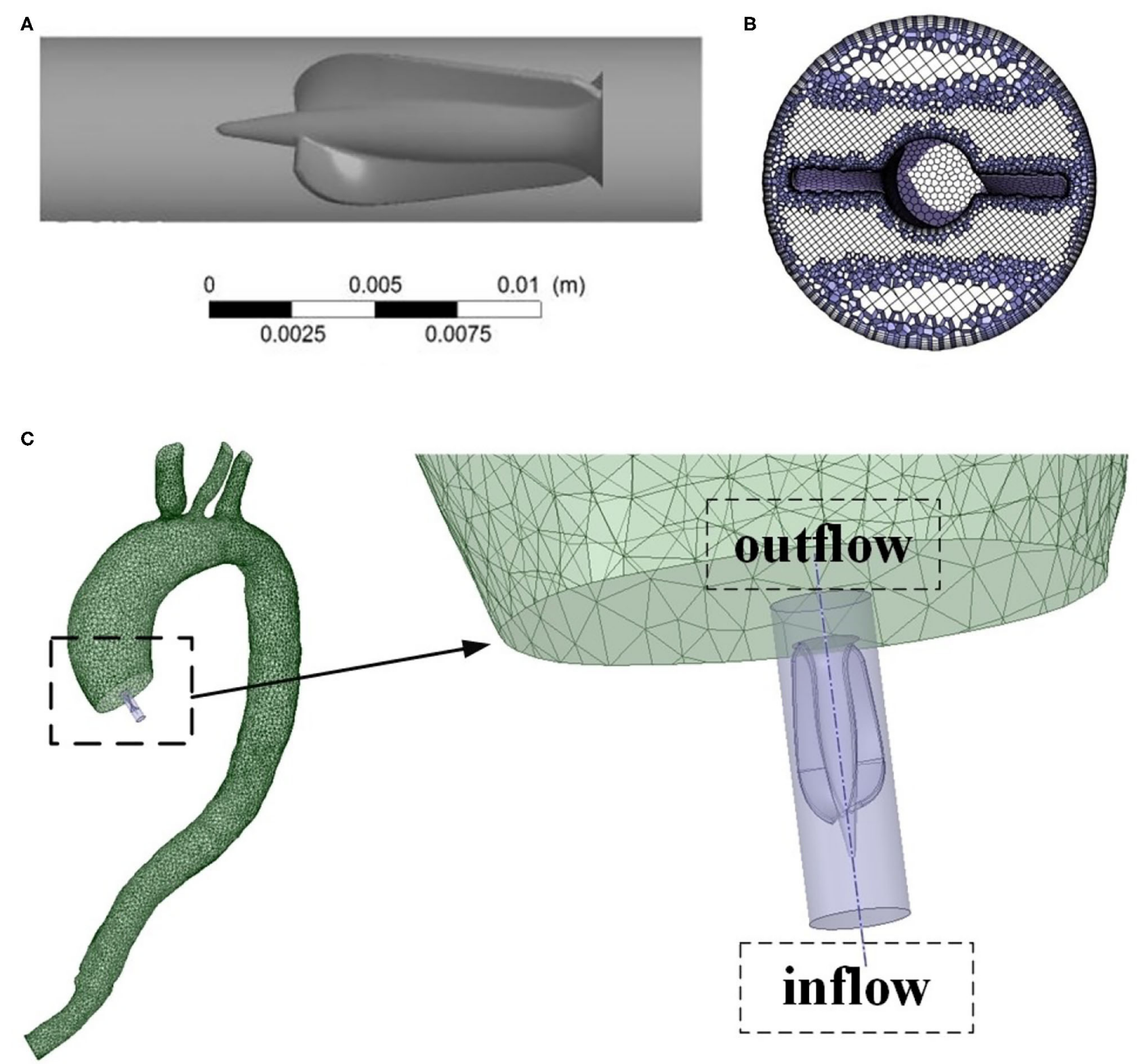

FIGURE 2 | (A) Geometric model of the Impella CP. (B) Crosssection mesh structure of Impella CP. (C) The location of Impella CP and its partially enlarged detail.

flow field of Impella CP, resulting in the domain tetrahedral unstructured meshes of about 290,000 elements. Pulsatile velocity and pressure are set as the boundary conditions at the inlet and outlet of Impella CP (Estrada et al., 2011; Roberts et al., 2020), respectively, as shown in Figures 3C,E. The impeller rotates at a constant speed of $46,000 \mathrm{r} / \mathrm{min}$ with a sliding mesh. Second, during one cardiac cycle, the total flow rate is calculated as the sum of the flow rate of the outlet of Impella CP (Figure 3F) and the native flow rate generated by the heart. The total flow rate is used to calculate the pulsatile velocity in the aorta (inlet boundary condition) using the inlet area of the aortic arch. The other settings are the same as those for the patient-specific aorta model without Impella CP.

\section{Numerical Schemes and Procedures}

The semiimplicit method for pressure linked equations pressurevelocity method was used to solve the above fluid governing equations. The spatial discretization schemes of gradient, pressure, and momentum are based on least square cell, standard, and second-order upwind, respectively. Aorta surfaces and walls of Impella CP are set to the no-slip condition. Moreover, in this study, two representative time points $t_{1}$ and $t_{2}$ (Figure 3C) (Chi et al., 2017) and two critical locations $A_{1}$ and $A_{2}$ (Figures 1B,C) 

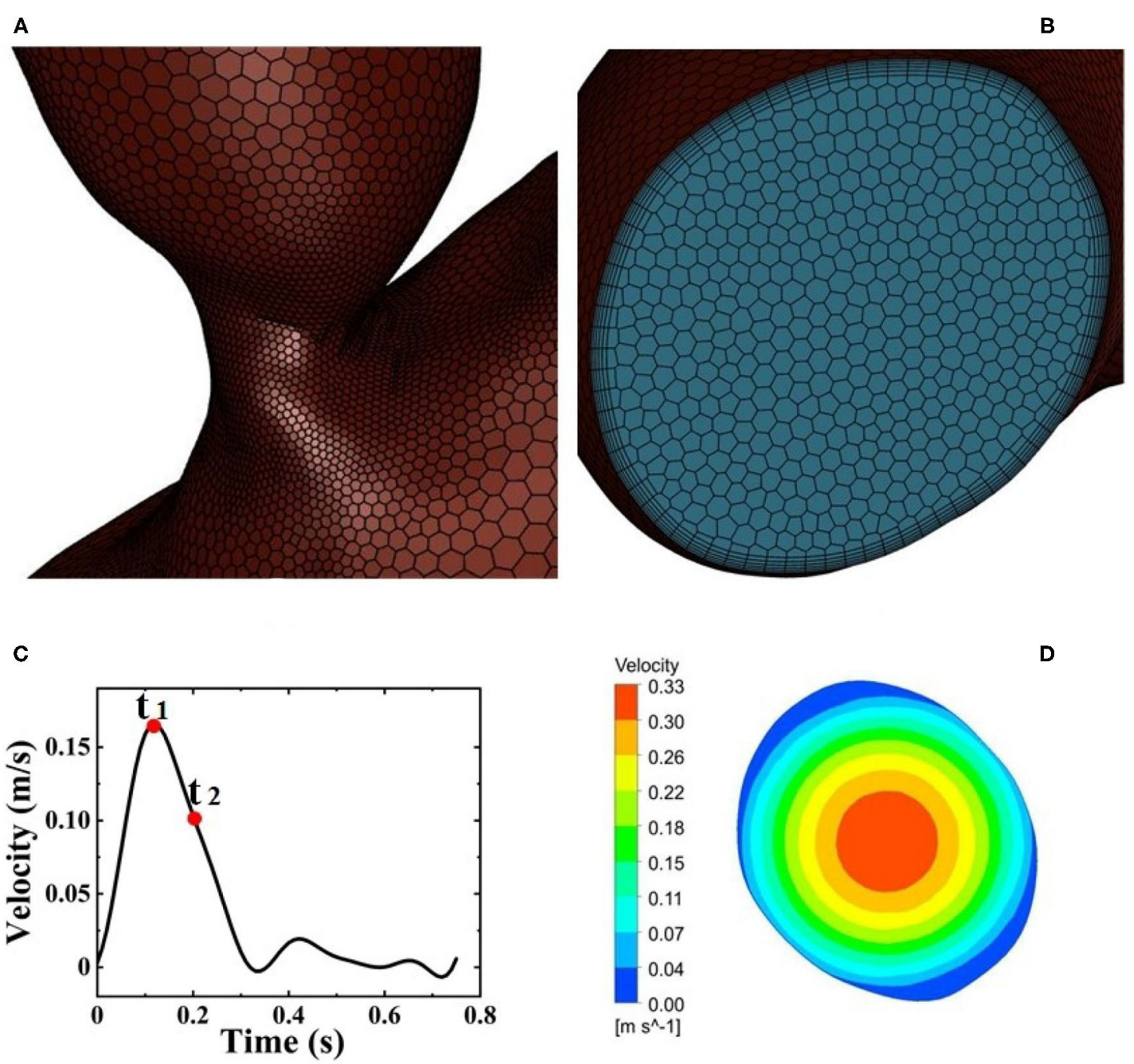

D

E
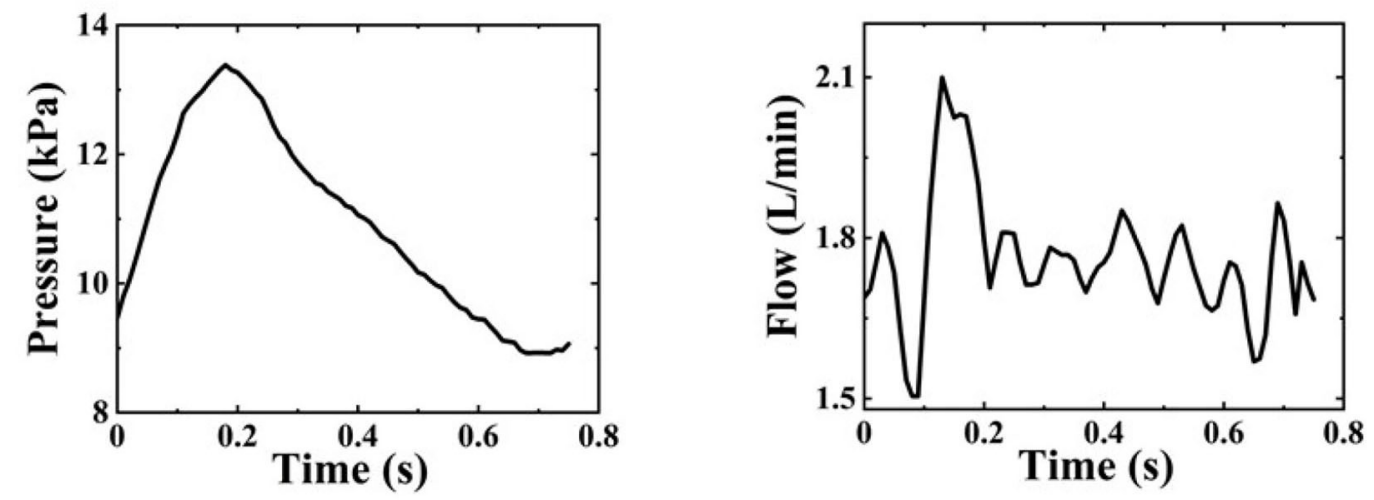

FIGURE 3 | (A) Local mesh structure of aortic arch. (B) Mesh structure of the inlet of aortic arch. (C) Pulsatile blood flow velocity at the inlet of aorta from one HF patient during one cardiac cycle and two selected critical time points $t_{1}$ and $t_{2}$. (D) Velocity distribution of fully developed blood flow perpendicular to the direction of blood flow. (E) Pulsatile pressure at the outlet of Impella CP. (F) Pulsatile flow rates at the outlet of Impella CP. 


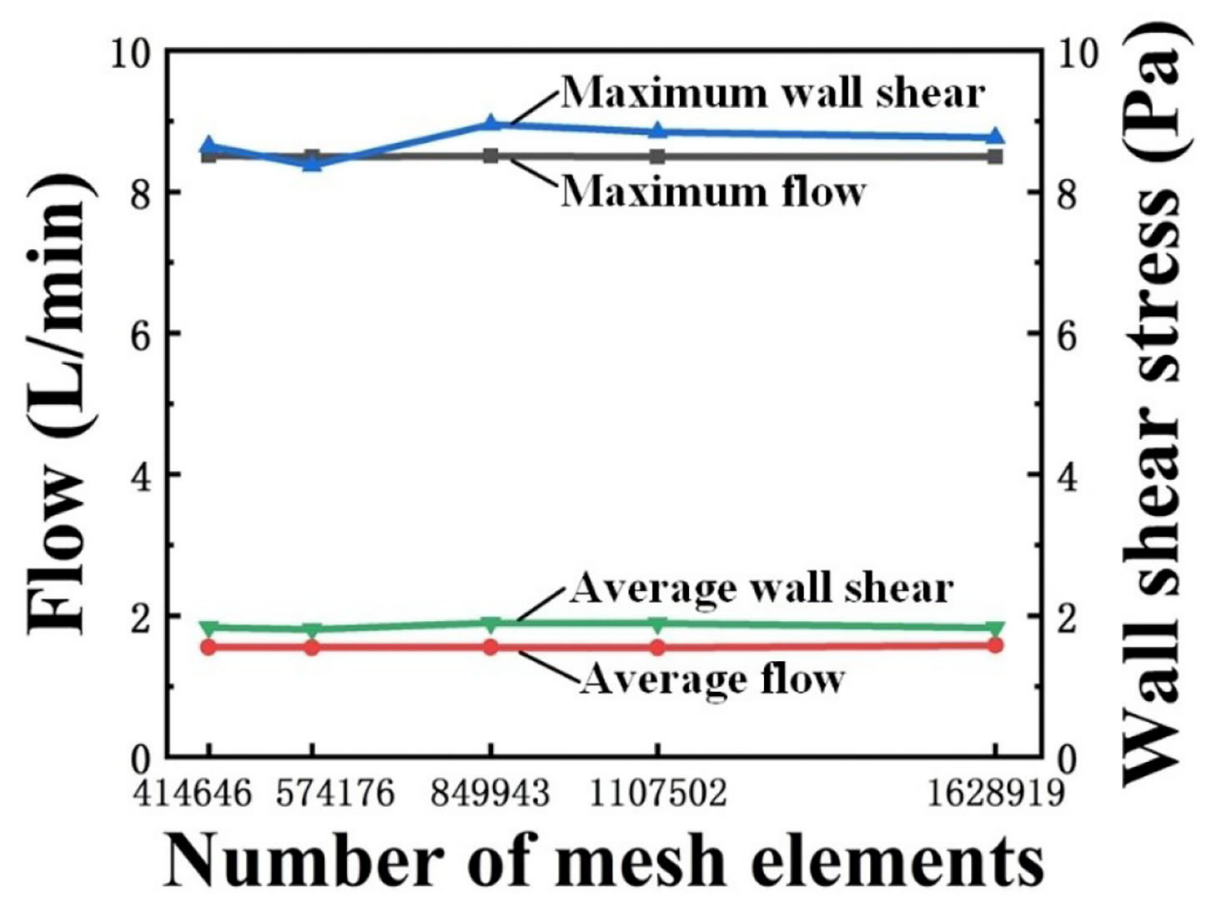

FIGURE 4 | Mesh independent verification results.

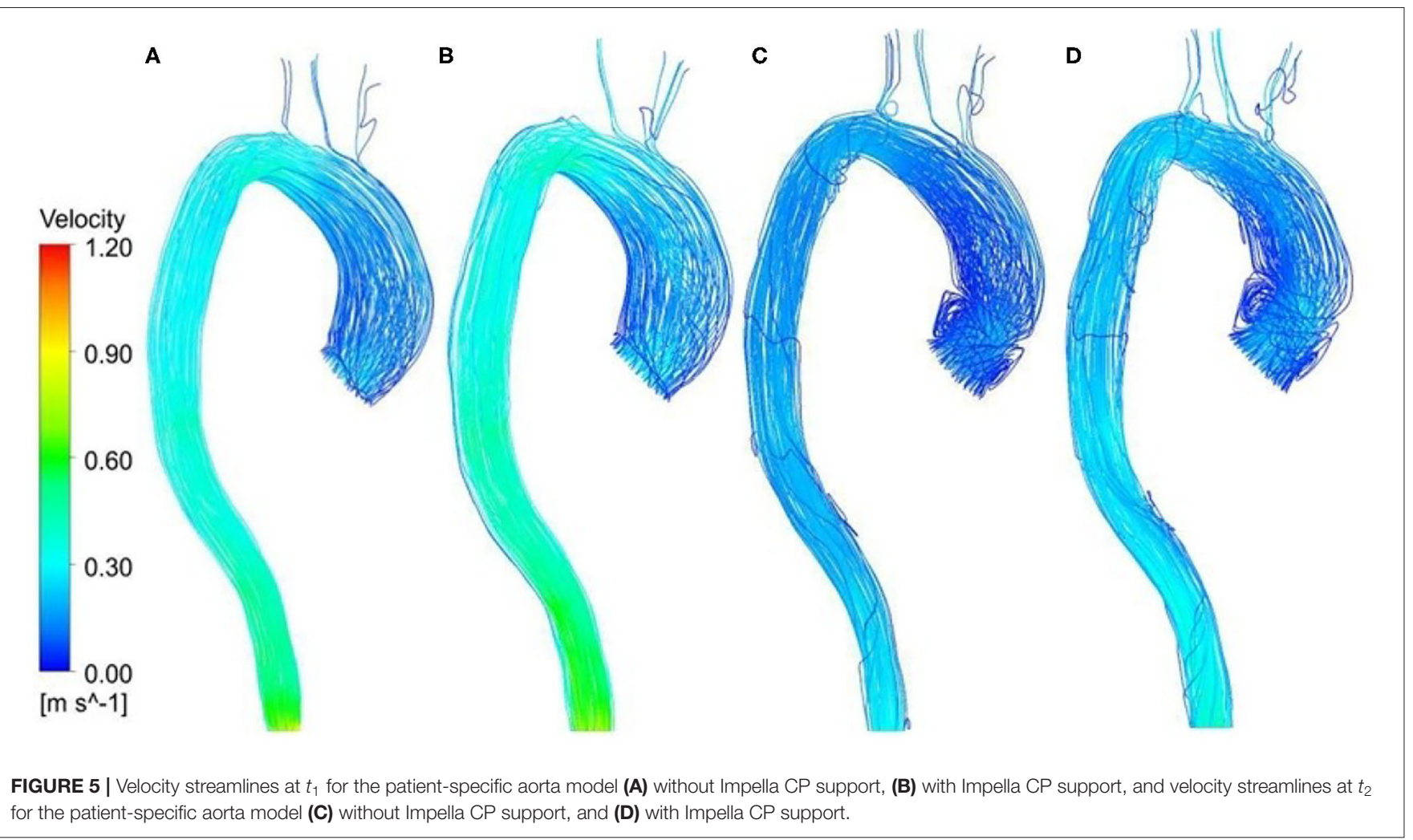

are selected for hemodynamic analysis. In detail, $t_{1}$ is chosen at $0.12 \mathrm{~s}$ as the peak systole, while $t_{2}$ is chosen at $0.22 \mathrm{~s}$ as the representative point during the deceleration ejection period. The locations $A_{1}$ and $A_{2}$ are near the ascending aorta and bifurcating area, respectively, because clinically the outflow of Impella CP is at the ascending aorta (near $A_{1}$ ) and the sharp geometric changes 

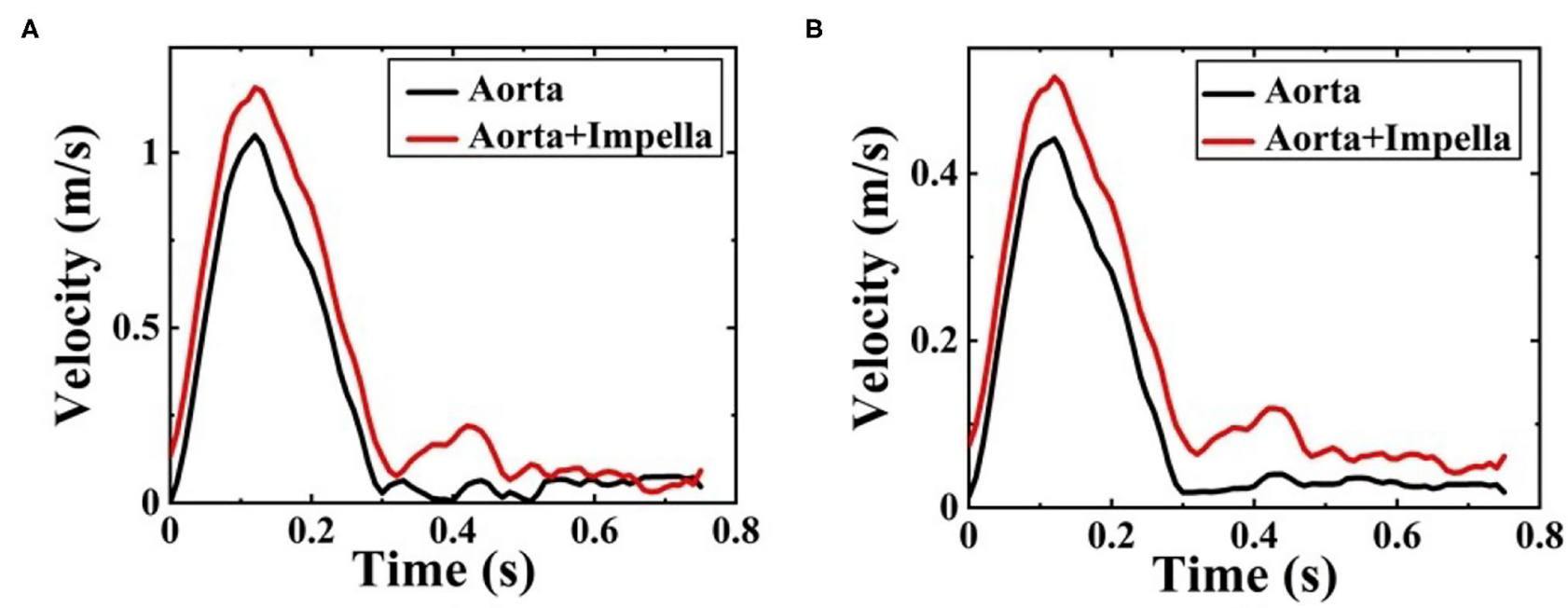

FIGURE 6 | (A) The center velocity at aortic arch outlet during one cardiac cycle without and with the Impella CP support and (B) the average velocity in the aortic arch during one cardiac cycle without and with the Impella CP support.

could easily happen at the bifurcating areas (near $A_{2}$ ). Some WSS and pressure waveforms were obtained using the "chart" function in CFD-post of ANSYS Fluent 2020 R1.

\section{Mesh and Time Independence Test}

Mesh independence test in this study was implemented by using five different meshes with 414,646; 574,176; 849,943; 1,107,502; and 1,628,919 elements, respectively. The convergence criterion was set as 0.0001 . The mesh had a negligible effect on the calculation results when the number of elements in the mesh was $>849,943$, as shown in Figure 4. Therefore, to optimize between computational cost and accuracy, 849,943-element mesh was selected.

The time steps were initially selected as 0.01 and $0.005 \mathrm{~s}$, respectively, for the time independence study. Under the two time-step conditions, the difference between the outlet velocity and WSS distribution was small $(<1 \%)$; however, the computational cost significantly increased when the time step of $0.005 \mathrm{~s}$ was used. As a result, a time step of $0.01 \mathrm{~s}$ was used in this study and the total calculation time was $30.5 \mathrm{~h}$. In addition, the simulations were conducted for six cardiac cycles to eliminate the initialization effect and achieve stable results. The data during the last cycle was used for analysis (Xiong et al., 2020).

\section{RESULTS}

\section{Flow Patterns}

Figures $\mathbf{5 A , B}$ shows a comparison of velocity streamlines during peak systole at $t_{1}$ for the patient-specific aorta model without and with the Impella CP assistance, respectively, and the velocity streamlines during the slow ejection period at $t_{2}$ for the patientspecific aorta model without and with the Impella CP assistance is shown in Figures $\mathbf{5 C , D}$, respectively. Apparently, the flows in this HF patient-specific aorta model were fairly organized. Obvious swirls were observed in the upstream of the aortic valve outlet during the slow ejection period corresponding to $t_{2}$ in Figures 5C,D. Figure 6A presents the center velocity at the aortic arch outlet during one cardiac cycle without and with the pump, respectively. The peak velocity is $1.05 \mathrm{~m} / \mathrm{s}$ without the pump compared with that of $1.19 \mathrm{~m} / \mathrm{s}$ with the pump. Figure 6B displays the average velocity in the aortic arch during one cardiac cycle without and with the Impella CP, respectively. The peak velocity is $0.44 \mathrm{~m} / \mathrm{s}$ without the Impella $\mathrm{CP}$ support and it increased to around $0.52 \mathrm{~m} / \mathrm{s}$ with the Impella $\mathrm{CP}$ support. In addition, the average aortic flow without the pump is $2.6 \mathrm{~L} / \mathrm{min}$, which is increased to $3.9 \mathrm{~L} / \mathrm{min}$ with the pump assistance.

\section{Wall Shear Stress}

The WSS distribution on the inner surface of the patient-specific aorta model at time $t_{1}$ and $t_{2}$ for one cardiac cycle without and with the Impella CP support is shown in Figure 7. Once the effects of Impella CP are considered, WSS values at most locations especially near the descending aorta and bifurcating areas would be larger than those without Impella CP support, regardless of the two selected representative time points. In addition, it is usually difficult to use an in vivo way to directly measure WSS, which is an extremely important hemodynamic quantity. Thus, in order to obtain the information of high WSS on the patient-specific aorta model, analyzing the WSS distribution patterns was conducted ahead of time. To more clearly observe the increased WSS in other regions, in this work, the maximum WSS value has been rescaled as decreased to $5.5 \mathrm{~Pa}$ and the upside of the aortic arch is enlarged, which is shown in Figure 7. Figure 8 shows the waveform of WSS at two selected locations $A_{1}$ and $A_{2}$. Clearly, the value of WSS without Impella CP support was lower than that with Impella CP support regardless of the two locations, and the maximum value of WSS at $A_{2}$ is much higher than that at $A_{1}$ regardless of the Impella CP support. 


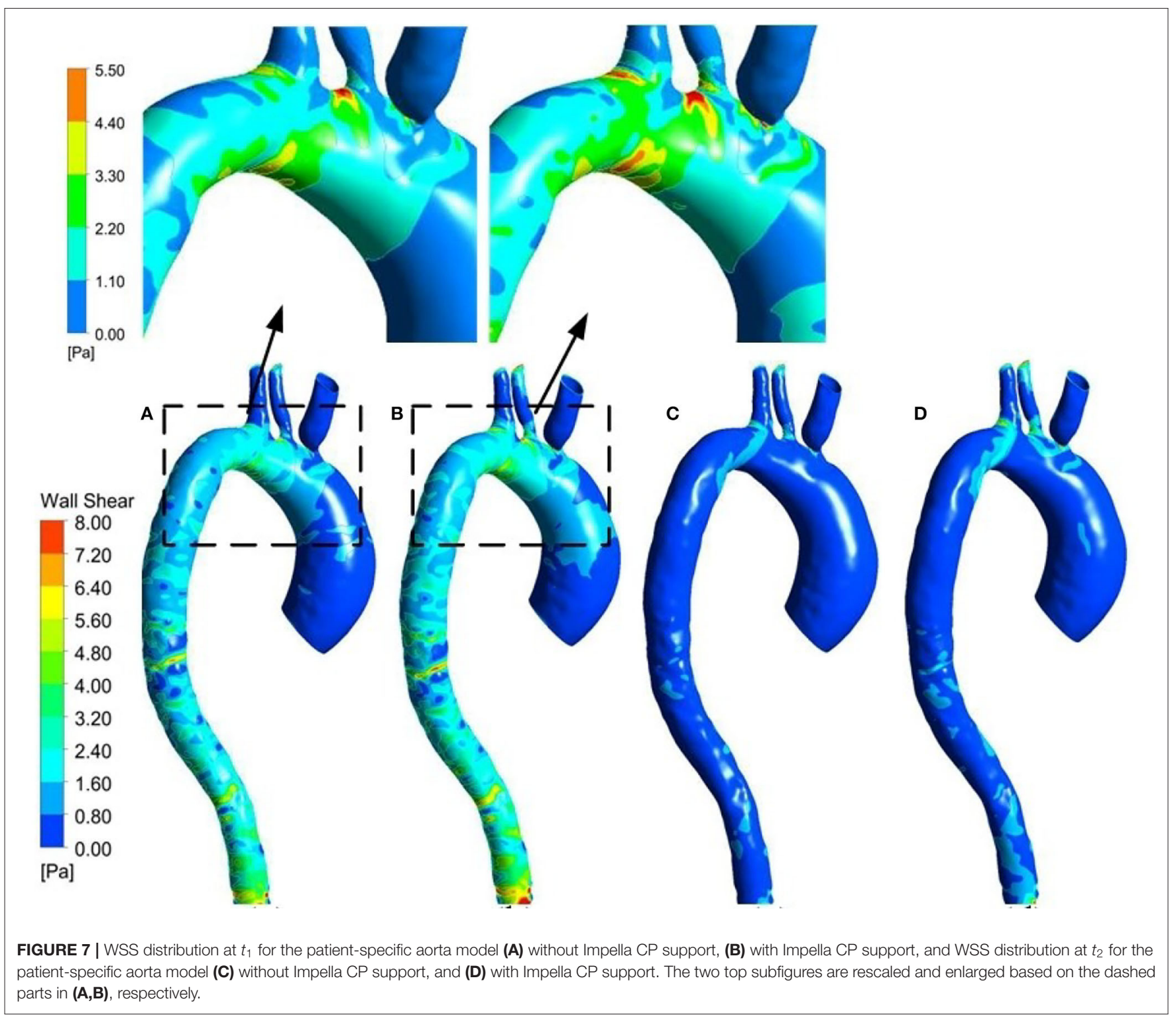

\section{$\Delta P$ Distribution}

In this study, $\Delta P$ means the difference between the pressure at any point on the vessel walls and the outlet pressure. Figures 9A,B shows the $\Delta P$ contour map of patient-specific aorta model at time $t_{1}$ during systolic period without Impella CP and during Impella CP support, respectively. $\Delta P$-values of the vessel wall are higher during Impella CP support than those without Impella $C P$ support, and $\Delta P$-values decrease gradually from the ascending aorta to the distal aorta. However, the opposite phenomenon was found at time $t_{2}$ during the deceleration ejection period without and with the Impella CP support, respectively, which is shown in Figures 9C,D such that $\Delta P$ values of vessel wall gradually increased from the ascending aorta to the distal aorta. Furthermore, Figure 10 shows $\Delta P$ waveforms at two selected locations $A_{1}$ and $A_{2}$. For $A_{1}$, the maximum $\Delta P$ value was $2.6 \mathrm{kPa}$ without Impella $\mathrm{CP}$ support and increased to $2.8 \mathrm{kPa}$, whereas the effects of Impella CP were considered. For
$A_{2}$, the maximum $\Delta P$-values were 2.4 and $2.6 \mathrm{kPa}$ without and with the Impella CP support, respectively. Note that in Figure 10, the two waveforms are extremely similar. The reason is that the distance between the selected two representative points $A_{1}$ and $A_{2}$ are very close, causing the pressure waveforms at $A_{1}$ and $A_{2}$ to be very similar.

\section{DISCUSSION}

Computational fluid dynamics simulation could effectively display the local hemodynamic effects in the cardiovascular system and enable assessments of the effects of LVADs in patients. In this numerical study, the proposed CFD modeling focused on the hemodynamic effects of a short-term Impella CP on a patientspecific aorta model, and was compared with the baseline results without Impella CP support. The numerical results demonstrated the changes inside the aorta, including flow velocity, WSS, and 
A

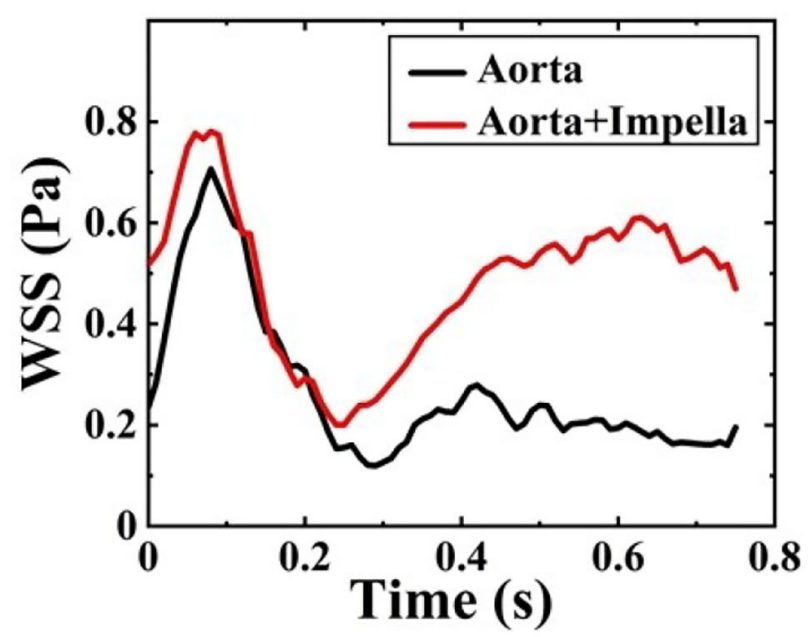

B

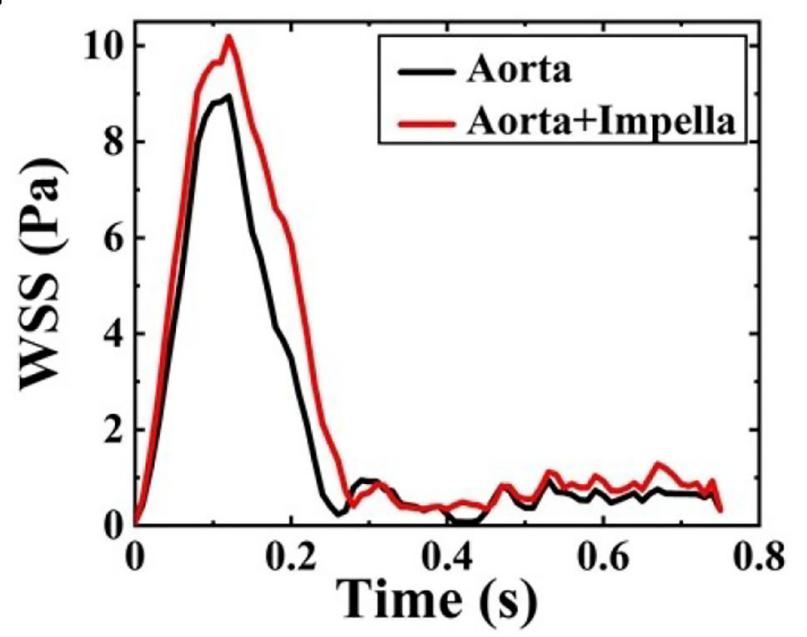

FIGURE 8 | Waveform of WSS at (A) $A_{1}$ and (B) $A_{2}$ during one cardiac cycle without and with Impella CP.

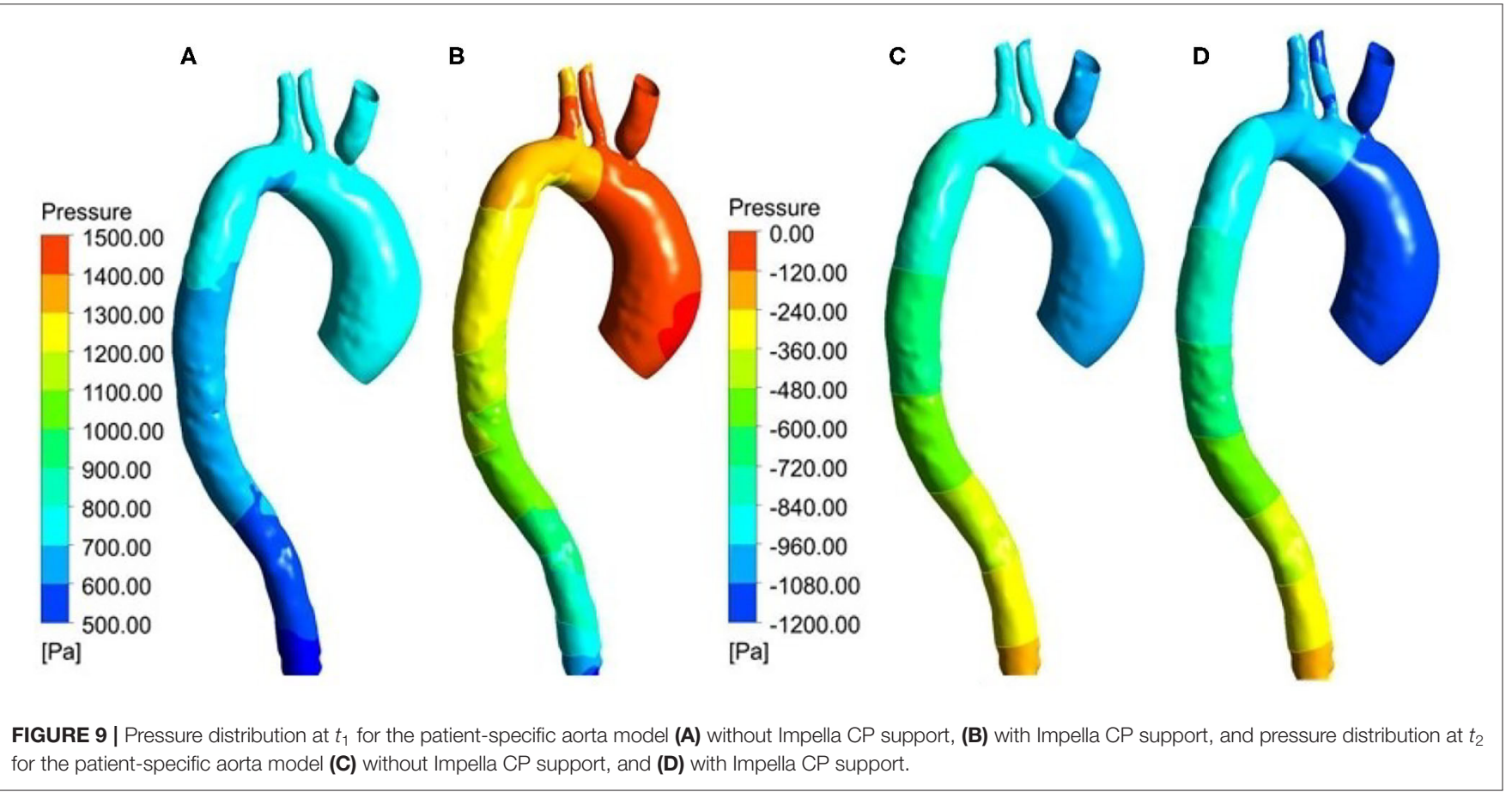

$\Delta P$. These data may help optimize the device and treatment of HF patients using short-term LVADs.

The disturbed flow that occurs near the inlet of aorta at $t_{2}$ in Figures 5C,D may be due to the slow velocity at this moment (Mazzitelli et al., 2016). Comparatively, such disturbed flow was not found in Figures 5A,B in the ascending aorta at $t_{1}$. The peak value of averaged velocity in the aortic arch in Figure 6B is lower than that of center velocity at aortic arch outlet in Figure 6A because the velocity is inversely proportional to the area. The center velocity at aortic arch outlet, the average velocity in the aortic arch, and the average aortic flow rates with the Impella CP support are higher than those without the Impella CP support.

In this CFD modeling, the boundary conditions used pulsatile velocity and pressure at the inlet and outlet of Impella $\mathrm{CP}$, respectively. However, the rotational speed of Impella $\mathrm{CP}$ was held constant. It has been proven that LVAD support diminished (nonphysiological) arterial pulsatility. Diminished pulsatility has been associated with adverse events in peripheral blood vessels and other organs, like arteriovenous malformation, hemorrhagic stroke, and damage to other organs such as kidney (Soucy 

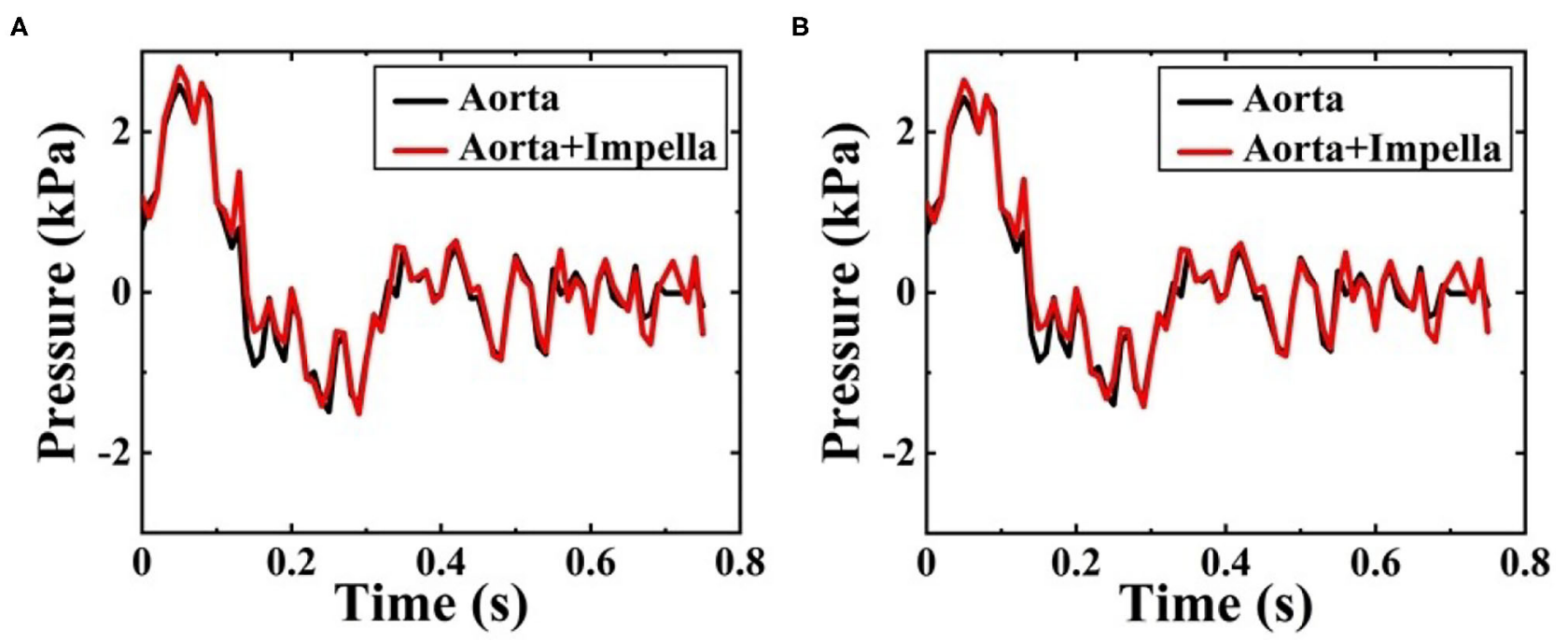

FIGURE 10 | Pressure waveforms at (A) $A_{1}$, and (B) $A_{2}$ during one cardiac cycle without and with the Impella CP assistance.

et al., 2013; Ross et al., 2018; Cho et al., 2019). Even short-term diminishment of pulsatility has been associated with endothelial dysfunction (Nguyen et al., 2021). In contrast, LVADs with pulsatile pump speed mode could improve arterial pulsatility and did not further lead to the risk of blood damage (Chen et al., 2018; Haglund et al., 2019). Therefore, one of the future works is to study the quantitative relationship between the modulated pump speed operation of short-term LVADs anastomosed to the patient-specific aorta model and key hemodynamic signals.

As one of the most important hemodynamic parameters, WSS is related to the vascular and arterial adaptation. The simulation results showed that changes in WSS with and without the Impella $\mathrm{CP}$ support at two selected representative locations $A_{1}$ and $A_{2}$ were obvious. WSS values on the aorta were much lower than those within the Impella CP. The highest shear stresses were generated at the impeller tips when the Impella CP is rotating at a very high speed $(\sim 46,000 \mathrm{rpm})$. Comparatively, WSS in the flow field is related to the blood velocity, which cannot be as high as that at the impeller tip. In general, reduction of WSS within the LVADs is beneficial as it reduces the potential risk of blood trauma, which remains an important clinical concern. In addition, with reference to the $\Delta P$ analysis, it is possible to note that $\Delta P$ was usually larger than WSS. Especially at $t_{1}$, the peak systole, blood increased and flowed via the ascending aorta with high $\Delta P$, which expanded the arterial wall due to the compliance of the aorta.

This study was still subject to some limitations. For example, these results were specific to one clinical patient-specific aorta data, which ensured that the individual effects of the LVAD support can be analyzed on a patient specific basis. However, global predictions of the LVAD support on aorta required averaging of aortic dimensions on a larger cohort of patients. The aortic model did not consider small aortic branch vessels distal to the aortic arch vessels that can cause additional outflows, and this CFD study did not analyze measures of hemolysis including hemolysis index. Moreover, in this research we only studied the changes in the local hemodynamic effects of aortic arch before and after implanting Impella $\mathrm{CP}$, and zero pressure was used as the outlet condition, implying that the characteristics of downstream vascular system of aortic arch were not considered in the CFD simulation by using the Windkesel model with boundary conditions of proximal resistance and capacitance, and distal resistance (Xiong et al., 2020), which may cause negative pressure and dramatic changes of $\Delta P$ in Figure 10. One of the future work is to reset the boundary conditions of outlet by considering the factors of downstream vascular system of aortic arch to make the CFD simulation results more consistent with the clinical results. Despite these limitations, the proposed study demonstrated the local hemodynamic changes in the aorta with short-term LVAD support devices.

\section{CONCLUSION}

In this paper, a quantitative CFD simulation was conducted for studying the local hemodynamic effects of a short-term LVAD in a patient-specific aorta model. Velocity, WSS, and $\Delta P$ in the aorta were calculated without and during the Impella CP support for comparison. Results demonstrated that Impella CP support augmented velocity, WSS, and $\Delta P$ within the aorta. Future study is needed using more effective and accurate CFD modeling, and with larger number of subjects and pulsatile pump speed conditions.

\section{DATA AVAILABILITY STATEMENT}

The original contributions presented in the study are included in the article/supplementary material, further inquiries can be directed to the corresponding author/s. 


\section{ETHICS STATEMENT}

Ethical review and approval was not required for the study on human participants in accordance with the local legislation and institutional requirements. Written informed consent for participation was not required for this study in accordance with the national legislation and the institutional requirements.

\section{AUTHOR CONTRIBUTIONS}

YW: methodology, writing-original draft preparation, and editing. JW: software and writing-original draft preparation.

\section{REFERENCES}

Amin, A. P., Spertus, J. A., Curtis, J. P., Desai, N., Masoudi, F. A., Bach, R. G., et al. (2020). The evolving landscape of Impella use in the United States among patients undergoing percutaneous coronary intervention with mechanical circulatory support. Circulation 141, 273-284. doi: 10.1161/CIRCULATIONAHA.119.044007

Benjamin, E. J., Blaha, M. J., Chiuve, S. E., Cushman, M., Das, S. R., Deo, R., et al. (2017). Heart disease and stroke statistics-2017 update: a report from the American heart association. Circulation 135, e146-e603. doi: 10.1161/CIR.0000000000000485

Brüning, J., Hellmeier, F., Yevtushenko, P., Kühne, T., and Goubergrits, L. (2018). Uncertainty quantification for non-invasive assessment of pressure drop across a coarctation of the aorta using CFD. Cardiovasc. Eng. Technol. 9, 582-596. doi: 10.1007/s13239-018-00381-3

Carnahan, S. R., Koenig, S. C., Sobieski, M. A., Schumer, E. M., Monreal, G., Wang, Y., et al. (2017). Efficacy of subcutaneous electrocardiogram leads for synchronous timing during chronic counterpulsation therapy. ASAIO J. 63, 134-138. doi: 10.1097/MAT.0000000000000498

Chen, Z., Jena, S. K., Giridharan, G. A., Sobieski, M. A., Koenig, S. C., Slaughter, M. S., et al. (2018). Shear stress and blood trauma under constant and pulsemodulated speed CF-VAD operations: CFD analysis of the HVAD. Med. Biol. Eng. Comput. 57, 807-818. doi: 10.1007/s11517-018-1922-0

Chi, Q., He, Y., Luan, Y., Qin, K., and Mu, L. (2017). Numerical analysis of wall shear stress in ascending aorta before tearing in type A aortic dissection. Comput. Biol. Med. 89, 236-247. doi: 10.1016/j.compbiomed.2017.07.029

Cho, S. M., Moazami, N., Katz, S., Bhimraj, A., Shrestha, N. K., and Frontera, J. A. (2019). Stroke risk following infection in patients with continuous-flow left ventricular assist device. Neurocrit. Care 31, 72-80. doi: 10.1007/s12028-018-0662-1

Deissler, R. G. (1984). Turbulent solutions of the equations of fluid motion. Rev. Mod. Phys. 56, 223-254. doi: 10.1103/RevModPhys.56.223

Doehner, W., Ural, D., Haeusler, K. G., Celutkiene, J., Bestetti, R., Cavusoglu, Y., et al. (2018). Heart and brain interaction in patients with heart failure: overview and proposal for a taxonomy. A position paper from the study group on heart and brain interaction of the heart failure association. Eur. J. Heart Fail. 20, 199-215. doi: 10.1002/ejhf.1100

Estrada, R., Giridharan, G. A., Nguyen, M. D., Roussel, T. J., Shakeri, M., Parichehreh, V., et al. (2011). Endothelial cell culture model for replication of physiological profiles of pressure, flow, stretch, and shear stress in vitro. Anal. Chem. 83, 3170-3177. doi: 10.1021/ac2002998

Febina, J., Sikkandar, M. Y., and Sudharsan, N. M. (2018). Wall shear stress estimation of thoracic aortic aneurysm using computational fluid dynamics. Comput. Math. Methods Med. (2018). 2018:7126532. doi: $10.1155 / 2018 / 7126532$

Gramigna, V., Caruso, M. V., Rossi, M., Serraino, G. F., Renzulli, A., and Fragomeni, G. (2015). A numerical analysis of the aortic blood flow pattern during pulsed cardiopulmonary bypass. Comput. Methods Biomech. Biomed. Engin. 18, 1574-1581. doi: 10.1080/10255842.2014.930136
JP and MH: software. ZY: data acquisition, validation, and formal analysis. GG: writing-reviewing and supervision. YL and KQ: writing-reviewing, editing, and supervision. All authors contributed to the article and approved the submitted version.

\section{FUNDING}

This work was supported, in part, by the National Natural Science Foundation of China (Grant Nos. 32071314 and 31971243), LiaoNing Revitalization Talents Program of China (Grant No. XLYC1807016), and National Institutes of Health R01 grant (Grant No. 1R01HL150346).

Guan, P., Gu, J., Song, Z., Wang, C., Hu, W., and Zhang, J. (2021). Left ventricular geometry transition in hypertensive patients with heart failure with preserved ejection fraction. Eur. J. Heart Fail. 8, 2784-2790. doi: 10.1002/ehf2.13349

Haglund, T. A., Rajasekaran, N. S., Smood, B., Giridharan, G. A., Hoopes, C. W., Holman, W. L., et al. (2019). Evaluation of flow-modulation approaches in ventricular assist devices using an in-vitro endothelial cell culture model. $J$. Heart Lung Transplant. 38, 456-465. doi: 10.1016/j.healun.2018.10.007

$\mathrm{Hu}, \mathrm{K} ., \mathrm{Hu}, \mathrm{T} ., \mathrm{Ma}, \mathrm{H}$., et al. (2020). ANSYS CFD Introduction Guide: Fundamentals and Applications of Computational Fluid Dynamics. Beijing: China Machine Press.

Keen, S. K., and Johnson, M. R. (2019). Unusual presentation of acute systolic heart failure caused by acquired post-traumatic aortic coarctation. Circ. Heart Fail. 12:e06267. doi: 10.1161/CIRCHEARTFAILURE.119.006267

Koshy, A. O., Gallivan, E. R., McGinlay, M., Straw, S., Drozd, M., Tom, A. G., et al. (2020). Prioritizing symptom management in the treatment of chronic heart failure. ESC Heart Fail. 7, 2193-2207. doi: 10.1002/ehf2.12875

Ky, B., French, B., Khan, A. M., Plappert, T., Wang, A., Chirinos, J. A., et al. (2013). Ventricular-arterial coupling, remodeling, and prognosis in chronic heart failure. J. Am. Coll. Cardiol. 62, 1165-1172. doi: 10.1016/j.jacc.2013.03.085

Leite, S., Cerqueira, R. J., Ibarrola, J., Fontoura, D., Fernández-Celis, A., Zannad, F., et al. (2019). Arterial remodeling and dysfunction in the ZSF1 rat model of heart failure with preserved ejection fraction. Circ. Heart Fail. 12:e005596doi: 10.1161/CIRCHEARTFAILURE.118. 005596

Li, W., Lin, Y., Wang, S., Zhou, S., Ju, J., Wang, X., et al. (2020). Extracellular superoxide dismutase is associated with left ventricular geometry and heart failure in patients with cardiovascular disease. J. Am. Heart Assoc. 9:e016862. doi: 10.1161/JAHA.120.016862

Mazzitelli, R., Boyle, F., Murphy, E., Renzulli, A., and Fragomeni, G. (2016). Numerical prediction of the effect of aortic left ventricular assist device outflow-graft anastomosis location. Biocybern. Biomed. Eng. 36, 327-343. doi: 10.1016/j.bbe.2016.01.005

McEwan, P., Darlington, O., McMurray, J. J. V., Jhund, P. S., Docherty, K. F., Böhm, M., et al. (2020). Cost-effectiveness of dapagliflozin as a treatment for heart failure with reduced ejection fraction: a multinational health-economic analysis of DAPA-HF. Eur. J. Heart Fail. 22, 2147-2156. doi: 10.1002/ejhf.1978

Menter, F. R. (1994). Two-equation eddy-viscosity turbulence models for engineering applications. AIAA J. 32, 1598-1605. doi: 10.2514/3.12149

Nagao, K., Taniguchi, T., Morimoto, T., Shiomi, H., Ando, K., Kanamori, N., et al. (2018). Acute heart failure in patients with severe aortic stenosis. Circ. J. 82, 874-885. doi: 10.1253/circj.CJ-17-0610

Nakamura, N. (1999). Peripheral vascular remodeling in chronic heart failure: clinical relevance and new conceptualization of its mechanisms. J. Card. Fail. 5, 127-138. doi: 10.1016/S1071-9164(99)9 0035-0

Nguyen, K. T., Donoghue, L., Giridharan, G. A., Naber, J. P., Vincent, D., Fukamachi, K., et al. (2021). Acute response of human aortic endothelial cells to loss of pulsatility as seen during cardiopulmonary bypass. Cells Tissues Organs. doi: 10.1159/000512558. [Epub ahead of print]. 
Osswald, A., Karmonik, C., Anderson, J. R., Rengier, F., Karck, M., Engelke, J., et al. (2017). Elevated wall shear stress in aortic type B dissection may relate to retrograde aortic type A dissection: a computational fluid dynamics pilot study. Eur. J. Vasc. Endovasc. Surg. 54, 324-330. doi: 10.1016/j.ejvs.2017.06.012

Perinajová R., Juffermans, J. F., Westenberg, J. J. M., van der Palen, R. L. F., van den Boogaard, P. J., Lamb, H. J., et al. (2021). Geometrically induced wall shear stress variability in CFD-MRI coupled simulations of blood flow in the thoracic aortas. Comput. Biol. Med. 133:104385. doi: 10.1016/j.compbiomed.2021.104385

Qiao, Y., Gui, H., and Liu, X. (2016). Analysis of three-dimensional numerical simulation methods for turbulent flow past circular cylinder. Hydrosci. Eng. 3, 119-125. doi: 10.16198/j.cnki.1009-640X.2016.03.016

Reddy, Y. N. V., Andersen, M. J., Obokata, M., Koepp, K. E., Kane, G. C., Melenovsky, V., et al. (2017). Arterial stiffening with exercise in patients with heart failure and preserved ejection fraction. J. Am. Coll. Cardiol. 70, 136-148. doi: 10.1016/j.jacc.2017.05.029

Roberts, N., Chandrasekaran, U., Das, S., Qi, Z., and Corbett, S. (2020). Hemolysis associated with Impella heart pump positioning: In vitro hemolysis testing and computational fluid dynamics modeling. Int. J. Artif. Organs 43:039139882090984. doi: 10.1177/0391398820909843

Rogers, J. G., Pagani, F. D., Tatooles, A. J., Bhat, G., Slaughter, M. S., Birks, E. J., et al. (2017). Intrapericardial left ventricular assist device for advanced heart failure. N. Engl. J. Med. 376, 451-460. doi: 10.1056/NEJMoa1602954

Ross, D. W., Stevens, G. R., Wanchoo, R., Majure, D. T., Jauhar, S., Fernandez, H. A., et al. (2018). Left ventricular assist devices and the kidney. Clin. J. Am. Soc. Nephrol. 13, 348-355. doi: 10.2215/CJN.04670417

Sayago, I., Domínguez-Rodríguez, F., Oteo-Domínguez, J. F., Gómez-Bueno, M., Segovia, J., and Alonso-Pulpón, L. (2015). Impella $\mathrm{CP}^{\circledR}$ circulatory support device as a bridge to heart transplantation: first experience in Spain. Rev. Esp. Cardiol. (Engl. Ed.) 68, 906-908. doi: 10.1016/j.recesp.2015.06.017

Schrage, B., Ibrahim, K., Loehn, T., Werner, N., Sinning, J. M., Pappalardo, F., et al. (2019). Impella support for acute myocardial infarction complicated by cardiogenic shock. Circulation 139, 1249-1258. doi: 10.1161/CIRCULATIONAHA.118.036614

Schumer, E. M., Black, M. C., Monreal, G., and Slaughter, M. S. (2016). Left ventricular assist devices: current controversies and future directions. Eur. Heart J. 37, 3434-3439. doi: 10.1093/eurheartj/ehv590

Shavik, S. M., Wall, S., Sundnes, J., Guccione, J. M., Sengupta, P., Solomon, S. D., et al. (2021). Computational modeling studies of the roles of left ventricular geometry, afterload, and muscle contractility on myocardial strains in heart failure with preserved ejection fraction. J. Cardiovasc. Transl. Res. doi: 10.1007/s12265-021-10130-y. [Epub ahead of print].

Simaan, M. A., Ferreira, A., Chen, S., Antaki, J. F., and Galati, D. G. (2009). A dynamical state space representation and performance analysis of a feedbackcontrolled rotary left ventricular assist device. IEEE Trans. Control Syst. Technol. 17, 15-28. doi: 10.1109/TCST.2008.912123

Slaughter, M. S., Rogers, J. G., Milano, C. A., Russell, S. D., Conte, J. V., Feldman, D., et al. (2009). Advanced heart failure treated with continuous-flow left ventricular assist devices. N. Engl. J. Med. 361, 2241-2251. doi: 10.1056/NEJMoa0909938

Soucy, K. G., Koenig, S. C., Giridharan, G. A., Sobieski, M. A., and Slaughter, M. S. (2013). Defining pulsatility during continuous-flow left ventricular assist device support. J. Heart Lung Transplant. 32, 581-587. doi: 10.1016/j.healun.2013.02.010

Van der Meer, P., Gaggin, H. K., and Dec, G. W. (2019). ACC/AHA versus ESC guidelines on heart failure: JACC guideline comparison. J. Am. Coll. Cardiol. 73, 2756-2768. doi: 10.1016/j.jacc.2019.03.478

Wang, Y., Koenig, S. C., Sobieski, M. A., Slaughter, M. S., and Giridharan, G. A. (2017). Hemodynamic benefits of counterpulsation, implantable, percutaneous, and intraaortic rotary blood pumps: an in-silico and in vitro study. Cardiovasc. Eng. Technol. 8, 439-452. doi: 10.1007/s13239-017-0314-1

Xiong, Z., Yang, P., Li, D., Qiu, Y., Zheng, T., and Hu, J. (2020). A computational fluid dynamics analysis of a patient with acute non-A-non-B aortic dissection after type I hybrid arch repair. Med. Eng. Phys. 77, 43-52. doi: 10.1016/j.medengphy.2019.10.019

Yoshida, S., Toda, K., Miyagawa, S., Yoshikawa, Y., Hata, H., Yoshioka, D., et al. (2020). Impact of turbulent blood flow in the aortic root on de novo aortic insufficiency during continuous-flow left ventricular-assist device support. Artif. Organs 44, 883-891. doi: 10.1111/aor.13671

Zhang, J., Chen, Z., Griffith, B. P., and Wu, Z. J. (2020). Computational characterization of flow and blood damage potential of the new maglev $\mathrm{CH}$ VAD pump versus the HVAD and HeartMate II pumps. Int. J. Artif. Organs 43, 653-662. doi: 10.1177/0391398820903734

Zhang, X., Luo, M., Fang, K., Li, J., Peng, Y., Zheng, L., et al. (2020). Analysis of the formation mechanism and occurrence possibility of post-stenotic dilatation of the aorta by CFD approach. Comput. Methods Prog Biomed. 194:105522. doi: $10.1016 /$ j.cmpb.2020.105522

Conflict of Interest: The authors declare that the research was conducted in the absence of any commercial or financial relationships that could be construed as a potential conflict of interest.

Publisher's Note: All claims expressed in this article are solely those of the authors and do not necessarily represent those of their affiliated organizations, or those of the publisher, the editors and the reviewers. Any product that may be evaluated in this article, or claim that may be made by its manufacturer, is not guaranteed or endorsed by the publisher.

Copyright (C) 2021 Wang, Wang, Peng, Huo, Yang, Giridharan, Luan and Qin. This is an open-access article distributed under the terms of the Creative Commons Attribution License (CC BY). The use, distribution or reproduction in other forums is permitted, provided the original author(s) and the copyright owner(s) are credited and that the original publication in this journal is cited, in accordance with accepted academic practice. No use, distribution or reproduction is permitted which does not comply with these terms. 